\title{
D'autant (plus) (que). De la corrélation comparative subjective à la cause emphatique intersubjective
}

Pascale Hadermann

Université de Gand, Belgique

\section{Introduction}

L'objectif de notre contribution est d'analyser les glissements de sens et d'emploi qui affectent la séquence d'autant (plus) que, séquence constituée de plusieurs éléments : la préposition de, l'adverbe de degré autant suivi éventuellement d'un autre adverbe de degré, tel que plus, moins ou mieux, et du morphème que nécessaire pour introduire le membre conséquent si celui-ci est exprimé :

(1) a. Je rêve souvent vaguement pendant tout ce temps et mes facultés en déclinent d'autant. Je sors toujours des séances dans un état de mobilité et d'incapacité d'attention ; ... (Maine de Biran 1814, $T L F i)$

(1) b. Donnez cent francs d'acompte, vous diminuerez d'autant la prochaine échéance. (Dict. Acad. Fr. ${ }^{9}$ )

(2) Si on lui objecte que ce préfet est protestant, il répond qu'il devait y aller d'autant plus. (Madelin ; Petit Robert)

(3) Pourquoi ne pas l'avoir acheté ? D'autant qu'il est bon. Je souhaiterais le garder pour le relire. Je vais l'acheter, d'ailleurs. (François, 2000) $)^{1}$

(4) La chaleur était suffocante, d'autant plus qu'on ne sentait pas [...] l'espace et le vent de la mer. (Daudet, Petit Robert)

1 Les exemples suivis du nom d'auteur et de l'année sont tirés de Frantext. Pour les autres illustrations, la source est toujours précisée. 
(5) des images [...] n'ayant voyagé qu'à l'intérieur de la mémoire, elles sont beaucoup plus stables et figées. Cela leur donne de la force et elles peuvent d'autant mieux chasser les autres, qui sont par nature plutôt mouvantes. (Roubaud, 2008)

(6) Je devais comprendre que nous n'en étions plus à trois jours près. Certes. Je le comprenais d'autant mieux que cette longue séparation avait eu des effets bénéfiques. (Groult, 2008)

(7) Rendre invisible dans le vocabulaire l'accession des femmes à de nouvelles fonctions, c'est une façon de la nier. Les hommes, eux, s'accommodent d'autant mieux du langage que les lacunes du vocabulaire ne les concernent pas. (Groult, 2008)

Comme l'illustrent les exemples (1) à (7), les éléments constitutifs de la séquence tantôt se suivent immédiatement, tantôt s'emploient de manière non contiguë (ex. (7)) ; ils peuvent maintenir leurs valeurs de base ou former une locution plus figée, susceptible de subir des glissements sémantiques de la comparaison vers l'expression de la cause. Avec cette coexistence de fonctionnements et de sens, qui nous permettra de formuler des hypothèses sur les facteurs déclencheurs des changements, nous nous situons au cœur de la problématique de la variation en français, les différentes réalisations illustrées ci-dessus étant encore toutes productives en français actuel.

Bien que constitué d'un ou de deux adverbes de degré dont les valeurs de base sont l'expression de la comparaison respectivement d'égalité (autant) et de supériorité (plus, mieux) ou d'infériorité (moins), la séquence est régulièrement appréhendée, dans les ouvrages de référence, comme une locution conjonctive exprimant un rapport causal (par exemple Grevisse et Goosse 2011 : 1139 b3, TLFi : cf. infra \$3.3) ${ }^{2}$. La question qui mérite d'être examinée est de savoir si et comment

2 Notons que la locution n'a pas attiré l'attention des chercheurs ; elle n'a été traitée que superficiellement dans le cadre d'études consacrées aux marqueurs comparatifs (récemment : Le Goffic 1993, Fournier et al. 2008, Kuyumcuyan 2008). Hadermann (à par.) la rattache à un ancien schème corrélatif inversé de type « morphème résomptif + morphème indéfini » où d'autant serait le résomptif et que (substitut de comme) la proforme indéfinie. 
d'autant (plus/moins/mieux) que - désormais d'autant (Mscal ${ }^{3}$ que -, qui morphologiquement parlant devrait orienter vers des effets comparatifs, parvient à exprimer des relations causales. Pour répondre à cette question, nous nous inspirerons d'approches centrées sur la grammaticalisation (cf. entre autres Traugott 1994, Giacalone Ramat et Hopper 1998, Prévost et Fagard 2007), la lexicalisation (cf. entre autres Lehmann 2002, Brinton et Traugott 2005, Prévost et Fagard 2007), la subjectification et l'intersubjectification (cf. entre autres Traugott 1989 et 2003, Degand et Fagard 2008), ainsi que d'approches qui consistent à établir un lien entre emploi de prédicats gradables ou scalaires ${ }^{4}$ et intersubjectivité que nous définissons comme : « a change which results in the development of meanings that explicitly reveal recipient design : the designing of utterances for an intended audience [...] at the discourse level » (Traugott et Dasher $2002: 31$ ).

Nous vérifierons plus spécifiquement dans quelle mesure d'autant (Mscal) que est affecté par les mécanismes suivants :

a) des processus de lexicalisation et de grammaticalisation

- la séquence formée de plusieurs éléments se figerait progressivement

- le figement aurait des répercussions sur le fonctionnement syntaxique et la séquence serait sujette à une ré-analyse

b) des processus de subjectification et d'intersubjectification

- la valeur de base de comparaison permettrait l'expression de la vision subjective du locuteur sur la projection scalaire des procès exprimés (cf. Athanasiadou 2007)

- de la comparaison de processus dériveraient des effets secondaires de type causal ; le locuteur tendrait à souligner l'importance de la cause introduite dans le but d'agir sur l'interlocuteur.

3 Mscal est l'abréviation de "marqueur scalaire », dénomination qui s'applique aux adverbes de degré référant à une échelle graduée (Hadermann et Inkova 2010).

4 Ces deux notions sont synonymes pour nous (cf. aussi Hadermann et Inkova 2010). 
Dans ce qui suit, nous décrirons brièvement notre méthodologie (section 2) avant de présenter les caractéristiques morphosyntaxiques et sémantiques de d'autant (Mscal) que (section 3) et d'étudier plus en détail la pertinence du trait $[+/- \text { co-variation }]^{5}$ pour les deux processus comparés et pour l'interprétation du rapport exprimé par d'autant (Mscal) que (section 4). Afin d'expliquer les tendances observées, nous vérifierons ensuite (section 5) dans quelle mesure notre séquence est touchée par des mécanismes tels que la lexicalisation ou la grammaticalisation.

\section{Données}

Etant donné que l'objectif de cette étude est de donner une description des différentes configurations possibles de la séquence d'autant (Mscal) que et des rapports qui les relient entre elles, nous nous sommes appuyée sur Frantext (documents à partir de 2000) et sur des ouvrages de référence (grammaires et dictionnaires du français moderne) pour récolter des occurrences révélatrices des divers cas de figure. Nous n'avons pas effectué de tests statistiques pour vérifier si les configurations montrent une prédilection pour tel ou tel type de registre, de genre ou de contexte en français actuel. Ceci pourra faire l'objet d'une recherche ultérieure centrée entre autres sur le paramètre " diaphasique ». Frantext nous a néanmoins fourni les fréquences relatives à partir du moyen français, que nous reproduisons dans les tableaux (1) et (2). Afin de donner une image correcte de l'importance relative de d'autant, nous mentionnons le nombre total de mots, suivi par le nombre total d'exemples en autant et par le nombre d'occurrences dans lesquelles autant apparait dans la séquence d'autant. Dans la colonne «\%», nous calculons l'emploi

5 La « co-variation » désigne ici toute liaison entre des variations affectant deux procès pour lesquels tout accroissement ou réduction de l'un déclenche un accroissement ou une réduction de l'autre (cf. <http://www.larousse.fr/dictionnaires/ francais $>$ ). 
relatif de autant par rapport au nombre total de mots et de d'autant par rapport à autant.

Tableau 1 : nombre et proportion des occurrences de d'autant par rapport à autant.

\begin{tabular}{|l|c|c|c|c|c|c|}
\hline & $\begin{array}{c}\text { Moyen } \\
\text { français }\end{array}$ & $\%$ & $\begin{array}{c}\text { Français } \\
\text { classique }\end{array}$ & $\%$ & $\begin{array}{c}\text { Français } \\
\text { moderne }\end{array}$ & $\%$ \\
\hline $\begin{array}{l}\text { Nombre de } \\
\text { mots }\end{array}$ & 6.121 .994 & & 856.568 & & 3.435 .347 & \\
\hline $\begin{array}{l}\text { Occurrences } \\
\text { de autant }\end{array}$ & 839 & $0,01 \%$ & 392 & $0,05 \%$ & 1.211 & $0,04 \%$ \\
\hline $\begin{array}{l}\text { Occurrences } \\
\text { de d'autant }\end{array}$ & 39 & $4,65 \%$ & 146 & $37,24 \%$ & 361 & $29,81 \%$ \\
\hline
\end{tabular}

Tableau 2 : français moderne - nombre et proportion des occurrences de d'autant par rapport à autant d'après le genre textuel.

\begin{tabular}{|l|c|c|c|c|c|c|}
\hline \multicolumn{7}{|c|}{ Français moderne } \\
\hline & Roman & $\%$ & Théâtre & $\%$ & Essai & $\%$ \\
\hline Nombre de mots & 1.042 .764 & & 190.613 & & 2.201 .970 & \\
\hline $\begin{array}{l}\text { Occurrences de } \\
\text { autant }\end{array}$ & 257 & $0,02 \%$ & 34 & $0,02 \%$ & 920 & $0,04 \%$ \\
\hline $\begin{array}{l}\text { Occurrences de } \\
\text { d'autant }\end{array}$ & 51 & $19,84 \%$ & 4 & $11,76 \%$ & 306 & $33,26 \%$ \\
\hline
\end{tabular}

Ces fréquences révèlent deux tendances intéressantes : (i) l'emploi croissant à partir du français classique et (ii) « l'essai » comme genre discursif préféré pour d'autant en français moderne ${ }^{6}$. Il faut donc retenir que ces formes sont relativement récentes et qu'elles semblent avant tout être exploitées dans des textes argumentatifs.

6 D'un point de vue diachronique, il serait intéressant de vérifier si cette préférence de la locution pour un genre dit «plus argumentatif » se manifeste également lors des stades antérieurs de la langue. 


\section{D'autant (Mscal) que - caractéristiques morphosyntaxiques et sémantiques}

\subsection{Constitution morphologique}

Les exemples (1) à (7) témoignent de la grande diversité dans les réalisations possibles pour d'autant (Mscal) (que). Ce qui mérite d'être souligné, c'est que le morphème que est le corrélatif de d'autant, même dans les cas où celui-ci est suivi d'un autre marqueur scalaire, comme mieux en (6) et (7), plus et moins en (8).

(8) a. Le sage est d'autant plus sage qu'il se soumet aux ordres de la provi-

$\curvearrowright$ dence. (Dict. Acad. Fr. ${ }^{1}$ )

(9) b. Il serait d'autant moins ridicule qu'il aurait eu moins de hâte à courir à sa déconvenue. (Romains, Grevisse et Goosse 2011)

En (8.a), la quantité véhiculée par autant est définie par se soumettre aux ordres de la providence et plus marque la supériorité de être sage :

(8) a'. Il est plus sage de combien, dans quelle mesure? — dans la mesure où il se soumet aux ordres de la providence.

A travers d'autant que la supériorité quantitative marquée par plus se trouve explicitée grâce au comparant de d'autant introduit par que. Il en va de même pour moins en (8.b) mais cette fois-ci il s'agit évidemment de l'explicitation de l'infériorité.

\subsection{Analyse syntaxique}

Pour ce qui est des caractéristiques syntaxiques de d'autant (Mscal) (que), il faut noter que la séquence ne se trouve pas antéposée dans l'énoncé et que, dans sa forme étendue (avec que), elle est soit intégrée, soit détachée par rapport à la phrase matrice ${ }^{7}$. Dans ce qui suit, nous

7 Dans notre corpus écrit, le détachement se marque par les signes de ponctuation. 
illustrerons ces deux modes de fonctionnement pour d'autant que (3) et pour d'autant Mscal que dans ses emplois contigus ou non (cf. d'autant Mscal que en (4) et (6) versus d'autant Mscal x que en (7) et (8)).

\subsubsection{D'autant que}

Dans cette réalisation, la locution semble préférer le fonctionnement détaché (3) et les attestations d'un éventuel fonctionnement intégré (9)-0 sont pour le moins ambiguës, du moins à l'écrit où nous n'avons pas les appuis désambiguïsants de l'intonation par exemple.

(3) Pourquoi ne pas l'avoir acheté ? D'autant qu'il est bon. Je souhaiterais le garder pour le relire. Je vais l'acheter, d'ailleurs. (François, 2000)

(9) Le mot me perce d'autant que, dans mes séquences, le « grabat » désigne la couche d'essai de mes figures asservies et joyeuses. (Guyotat, 2006)

(10) Inutile de tergiverser d'autant que l'éléphant en stand-by peut à tout moment barrir. (Verheggen, 2004)

En (9), d'autant pourrait porter sur le verbe de la première prédication : « me percer de combien ? - de autant », percer acceptant en effet la quantification :

(11) Arnaud le perce de trois balles. (Beauchamp $1806 T L F i$ )

2

En revanche, la quantification de tergiverser en 0 semble moins facilement acceptable - «? tergiverser de combien ? - et la portée de la proposition introduite par d'autant que est plutôt de type exophrastique (cf. Guimier 1996 ${ }^{8}$ ).

8 Cf. Guimier (1996) : la portée est exophrastique lorsqu'elle prend pour objet l'énonciation d'une phrase. Elle s'oppose à la portée endophrastique qui caractérise des constituants internes à la phrase et qui se subdivise à son tour en portée intraprédicative - au sein du prédicat - et extraprédicative - hors prédicat (cf. les compléments de phrase); voir aussi Fuchs et Fournier (2007). Dans notre étude, seule l'opposition endo-/exophrastique sera prise en compte. 


\subsubsection{D’autant Mscal (x) que}

Accompagnée d'un second marqueur scalaire, la séquence accepte les fonctionnements intégré, (8)-(12) (cf. aussi l'exemple (7)), et détaché (13)-(14) :

(8) Le sage est d'autant plus sage qu'il se soumet aux ordres de la providence.

(9) 2 ict. Acad.Fr.') d'offrir sa vie en même temps que la mienne, en même temps que moi, ça

2 mais personne ne l'avait fait. (Angot, 2006)

(10) L'abstinence ne fut pas trop difficile : la fièvre créatrice qui le dévorait la compensait largement, d'autant plus que ce qu'il écrivait était censé le (D) rocher d'elle. (Angot, 2006)

(11) Mais les mathématiques se remplissent pour moi de termes de plus en plus hostiles, d'autant plus menaçants que je m'y sens là au bord d'une réalité et d'une vérité qui m'aideraient à comprendre un autre courant du monde, une autre structure de la pensée, à me connaître moi-même, ... (Guyotat, 2007)

En (8) et (12), la valeur comparative est encore clairement présente : à travers d'autant que la supériorité quantitative marquée par plus se trouve explicitée - « il est plus sage d'un degré qui est égal à sa soumission » en (8) et " je l'admirais plus d'un degré qui est égal à sa présence » en (12) -, alors qu'en (13) elle s'estompe - le premier effet de sens n'étant pas « la fièvre la compensait plus largement d'un degré qui est égal au rapprochement ». Cette perte de la valeur comparative va de pair avec un figement progressif de la locution lorsqu'elle est utilisée de manière contiguë en tête de phrase (cf. infra) et il se produit un glissement de sens : d'autant Mscal que introduit une cause spécifique pour le procès exprimé dans la phrase matrice : « (...) la fièvre créatrice compensait largement l'abstinence, parce que ce qu'il écrivait était censé le rapprocher d'elle ». Quant à l'exemple (14), la proposition en d'autant Mscal x que est jointe à la phrase matrice comme une prédication seconde et l'ensemble en d'autant que $Q$ sert à préciser le degré véhiculé par plus menaçants. 


\subsection{Effets sémantiques}

Nous venons de voir que dans certains emplois l'effet de sens comparatif de d'autant (Mscal) que cède la place à d'autres nuances sémantiques et la question se pose de savoir comment ce glissement se trouve reflété dans des ouvrages de référence tels que le Trésor de la Langue française (TLFi). Ce dictionnaire traite la séquence sous l'entrée « autant », lexème pour lequel il prévoit trois emplois :

a) « adverbe de comparaison exprimant en phrase comparative, explicite ou implicite, l'égalité de deux procès, quant à leur intensité, leur quantité ou leur valeur»

b) « adverbe exprimant un rapport de grandeur, d'intensité entre deux procès, la notion d'égalité passant au second plan, ou s'effaçant »

c) «adverbe d'intensité en composition dans les locutions adverbiales ou conjonctives »

D'autant (Mscal) (x) (que) est repris en (b) et (c). Sous (b) figurent des exemples avec et sans que; la séquence y serait synonyme de respectivement «dans cette proportion » (15) et « dans la même proportion que », « (surtout) dans la mesure où » (16) :

(15) Augmenter une somme d'autant. (TLFi)

$=$ Augmenter dans la même proportion $>$ doubler la somme

(16) Il est sûr qu'un verre d'alcool peut changer toute une vie. Et je dis même qu'il la jettera d'autant plus bas qu'elle aura plus de germes de noblesse. (Alain 1909, TLFi)

$=$ L'alcool jettera la vie plus bas dans la mesure où elle aura plus de germes de noblesse.

En (c) les exemples sont tous accompagnés de que mais sémantiquement le TLFi distingue les séquences sans marqueur scalaire de celles avec marqueur scalaire, les premières ayant le sens de « vu que », « attendu que » (17), les secondes signifiant « surtout parce que », « surtout du fait que » (18).

(17) Mais me feriez-vous le plaisir de venir demain poursuivre cet intéressant entretien? D'autant que la dactylographe qui travaille dans mon bureau entasse les fautes de frappe, et que je songe à l'écarter. (Giraudoux 1942, TLFi) 
(18) ... enfin, coquette, ambitieuse, envieuse, fausse et vindicative, elle est d'autant plus dangereuse, que son ton, sa vivacité, ses manières si naturelles, son air ouvert, étourdi, et jusqu'à sa gaieté, ne permettent pas de la soupçonner d'artifices, ... (de Genlis 1795, TLFi)

C'est donc dans la rubrique (c) que le glissement vers les valeurs causales est le plus notable. En (18), il ne s'agit pas seulement d'une comparaison entre les deux processus de l'énoncé ; d'autant plus que y sert à introduire une cause particulière pour « être dangereuse », c'est-à-dire « ne pas permettre de la soupçonner d'artifices ».

Le traitement séparé des exemples (16) en (b) - avec maintien de la valeur de base - et (18) en (c) - avec développement d'un sens causal se justifie par des indices formels : la présence d'un marqueur scalaire plus dans la sous-phrase en (16), par opposition à l'apparition d'un prédicat nié et non gradable - « ne pas permettre »-en (18). Ces indices nous permettront de mieux cerner les raisons pour lesquelles la séquence d'autant (Mscal) que, qui morphologiquement parlant devrait orienter vers des effets comparatifs, en arrive à exprimer des relations causales et de mieux identifier la nature de ces relations causales. Dans ce qui suit, nous retracerons les glissements qui affectent la séquence d'autant (Mscal) (que) et nous vérifierons l'hypothèse du passage du rapport comparatif à l'expression de la cause privilégiée, fondée sur un rapport d'(inter)subjectivité.

\section{Du rapport comparatif au rapport causal}

Comme nous l'avons déjà noté, la séquence en d'autant Mscal que (donc la séquence dans sa forme la plus étendue) met souvent en rapport deux prédications gradables ou scalaires entre lesquelles s'établit un rapport comparatif avec parfois une nuance de type co-variationnel. Dans ce qui suit, nous étudierons la pertinence du trait [+/- co-variation] pour décrire les effets sémantiques véhiculés par d'autant Mscal que, ainsi que l'importance des propriétés sémantiques des prédications liées. 


\section{$4.1+$ co-variation entre les deux processus}

(16) Il est sûr qu'un verre d'alcool peut changer toute une vie. Et je dis même qu'il la jettera d'autant plus bas qu'elle aura plus de germes de noblesse. (Alain 1909, TLFi)

En (16), il s'établit un rapport de co-variation scalaire entre avoir des germes de noblesse et jeter bas; les deux prédicats sont projetés chacun sur une échelle et le locuteur les relie proportionnellement (16.a), comme dans une corrélative comparative (16.b) :

(16) a. Il la jettera plus bas à un degré qui est égal au fait d'avoir plus de germes de noblesse

(16) b. Plus elle aura des germes de noblesse, plus bas il la jettera.

Par le biais de d'autant plus que il exprime son point de vue subjectif quant au degré du processus jeter bas, qu'il met en corrélation avec avoir des germes, procès également quantifié à l'aide du marqueur scalaire plus. Notons que l'implication du locuteur à son énoncé est mise en évidence par et je dis même. Nous définissons ce type de covariation comme étant l'expression d'une «scalarité corrélative, de type subjectif ». De plus, cette scalarité corrélative s'accompagne d'une connotation causale. La co-variation implique en effet que la variation d'un paramètre $x$ produit un effet sur le paramètre $y$ (Hadermann et al. 2010); la variation de $x$ sera donc la cause du changement en $y$ (cf. entre autres Bertocchi et Maraldi 2010 : 93, pour des exemples latins) et ainsi, nous passons insensiblement à la causalité $(16 . c)^{9}$ :

9 La relation d'implication entre les deux propositions dans une structure corrélative comparative est souvent considérée comme étant proche de celle des structures conditionnelles en protase-apodose (cf. entre autres Beck 1997, Bertocchi et Maraldi 2010, Sanchez 2010, Inkova et Hadermann 2013) :

« [...] la sémantique de ces constructions ressemble à celle des conditionnelles dans la mesure où il est possible de reconstruire, pour les comparatives corrélatives, une relation d'implication unidirectionnelle si $P$ au grade $g>Q$ au grade g'. » (Sanchez 2010 : 129)

Ce schéma interprétatif souligne l'existence du rapport sous-jacent de type cause-conséquence. 
(16) c. Comme elle aura plus de germes de noblesse, il la jettera encore plus bas.

\section{$4.2 \pm$ co-variation entre les deux processus}

(8) Le sage est d'autant plus sage qu'il se soumet aux ordres de la providence.

En (8), le prédicat, être sage, est projeté sur une échelle et le locuteur relie le degré de sagesse au processus de la sous-phrase, c'est-à-dire à la soumission (8.a) ou, éventuellement, au « degré » de soumission (8.b). En effet, même si la sous-phrase ne contient pas de marqueur explicite de scalarité, il existe une possibilité de double lecture qui provient de la nature scalaire du prédicat ${ }^{10}$ se soumettre aux ordres de la providence :

(8) a. Comme il se soumet aux ordres de la providence, il sera encore plus sage.

(8) b. Comme il se soumet plus aux ordres de la providence, il sera encore plus sage.

Le processus sous-phrastique fournit « l'explication » du haut degré attribué au processus de la phrase matrice, ce qui déclenche la nuance de causalité. Nous sommes à nouveau en présence de l'expression d'une scalarité corrélative, de type subjectif.

\section{3 - co-variation entre les deux processus}

(12) ... je l'admirais d'autant plus qu'il était là,... (Angot, 2006)

En (12), être là n'étant pas un prédicat scalaire, il est impossible de qualifier le rapport entre les deux procès de co-variationnel. La quantification porte uniquement sur admirer et, avec la perte de la co-variation, l'effet comparatif s'estompe (12.a). Le locuteur se limite à souligner le

10 Pour la corrélative comparative en latin, Bertocchi et Maraldi (2010 : 94-95) ont également montré l'impact, sur l'interprétation de l'énoncé, de la présence ou non d'un marqueur ou d'un prédicat scalaire dans la deuxième proposition. 
haut degré du procès de la phrase matrice et à en citer la cause dans la sous-phrase sans quantifier celle-ci (12.b).

(12) a. $\neq$ Je l'admirais plus d'un degré qui est égal au degré de sa présence.

(12) b. = Je l'admirais encore plus parce qu'il était là.

La sous-phrase en d'autant plus que a uniquement pour but de mieux convaincre l'interlocuteur de la vérité du procès de la phrase matrice ; d'autant plus que sert de marqueur d'une relation que nous appellerons « causalité intersubjective emphatique ».

Cette causalité emphatique s'appuie parfois sur d'autres types de rapports, tels que le contraste. En (18) se manifeste une opposition entre son ton, sa vivacité, ses manières si naturelles, son air ouvert, étourdi, et jusqu'à sa gaieté - sujet de la sous-phrase - et coquette, ambitieuse, envieuse, fausse et vindicative - appositions dans la phrase matrice ; en effet, l'on ne s'attend pas à ce que quelqu'un qui a l'air vif, naturel, ouvert, ... soit en même temps coquet, ambitieux, envieux, ...

(18) ... enfin, coquette, ambitieuse, envieuse, fausse et vindicative, elle est d'autant plus dangereuse, que son ton, sa vivacité, ses manières si naturelles, son air ouvert, étourdi, et jusqu'à sa gaieté, ne permettent pas de la soupçonner d'artifices, ... (de Genlis 1795, TLFi)

A travers une cause inattendue et contrastée, l'attention de l'interlocuteur est portée sur le danger dont le locuteur souligne le haut degré. D'autant plus que devient ici marqueur d'une «causalité intersubjective contrastée $\gg{ }^{11}$.

L'absence de co-variation caractérise également les énoncés dans lesquels la sous-phrase en d'autant plus que se trouve détachée de la

11 Un des relecteurs anonymes souligne à juste titre que ce type de causalité s'accompagne d'une nuance concessive, rapport qui s'appuie également sur des effets d'inattendu :

Même si elle est coquette, ambitieuse, envieuse, fausse et vindicative, elle est dangereuse, surtout dans la mesure où son ton, sa vivacité, ses manières si naturelles, son air ouvert, étourdi, et jusqu'à sa gaieté, ne permettent pas de la soupçonner d'artifices. 
phrase matrice et où la portée est de nature exophrastique. Nous pourrions en (12) avoir un détachement, ce qui donnerait (19) :

(19) ... je l'admirais, d'autant plus qu'il était là, ...

Dans ce type d'exemples, d'autant plus se rapporte au contenu de la sous-phrase. Il n'est plus question de co-variation mais bien d'une cause située sur une échelle à un point supérieur par rapport à une autre cause :

(19) a. Situation : je l'admirais

(19) b. Cause explicitée : il était là

(19) c. Cause non exprimée moins pertinente (par ex. il m'avait écrit)

(19) d. Sens : « Je l'admirais, surtout parce qu'il était là »

D'autant plus que sert ici à cadrer une cause que le locuteur privilégie et qu'il présente à l'interlocuteur comme se rapprochant beaucoup de la cause archétypale : le procès il était là se trouve projeté sur une échelle de causes possibles, d'où un effet de « causalité scalaire » reflétant l'évaluation subjective du locuteur avec une intention d'intersubjectification.

\subsection{Du rapport comparatif au rapport causal : bilan}

Le trait [+/- co-variation] s'est révélé extrêmement pertinent pour décrire les glissements de sens en jeu. Dans le cas de la présence du trait [+ co-variation], les deux prédications mises en relation sont gradables et la sous-phrase comporte un marqueur scalaire. La portée de d'autant Mscal que est de type endophrastique (fonctionnement intégré) et la valeur de base, comparative, reste présente, même si elle s'accompagne de nuances causales. Dans les cas de [ \pm co-variation], les deux prédicats sont gradables mais la sous-phrase ne comporte plus de marqueur scalaire. La portée reste de type endophrastique et la valeur comparative est bien présente quoique l'effet causal se fasse plus explicite : « encore plus ... parce que ». Nous avons donc à faire à un premier affaiblissement des valeurs de base avec une légère perte 
de l'autonomie de d'autant et du second marqueur scalaire (plus). Lorsque le trait [co-variation] est absent, ce marqueur scalaire ne joue plus aucun rôle dans la phrase matrice. La locution assume une portée de type exophrastique et elle apparaît uniquement dans des réalisations contiguës. Le rapport entre les deux procès est de nature causale scalaire, avec le sens de « surtout parce que ». Nous sommes ici en présence d'un début de figement et de réanalyse.

Ensemble avec la portée endo- ou exophrastique, le trait [+/co-variation] a en outre permis de mieux cerner la nature du rapport causal, avec un jeu sur le subjectif et l'intersubjectif : lorsque la portée est endophrastique, nous avons observé les trois cas de figure :
a. + co-variation : scalarité corrélative, subjective
b. \pm co-variation : scalarité corrélative, subjective avec effet causal
c. - co-variation : causalité emphatique ou contrastée

En revanche, dans le cas de la portée exophrastique, nous sommes toujours en présence du trait [- co-variation] et l'effet sémantique qui se dégage de la construction est celui d'une causalité scalaire, subjective avec intersubjectification ${ }^{12}$.

12 Cette analyse, illustrée pour d'autant plus (x) que, s'applique également aux séquences en d'autant mieux que, mieux étant une forme synthétique pour plus bien, et en d'autant moins que :

a. portée endophrastique

- + co-variation : scalarité corrélative, subjective (marqueur scalaire dans $Q$ ) Il serait d'autant moins ridicule qu'il aurait eu moins de hâte à courir à sa déconvenue. (Romains, Grevisse et Goosse 2011)

- \pm co-variation : scalarité corrélative, subjective avec effet causal (prédicat scalaire dans $Q$ )

Je n'avais pas envie de rire. Je riais d'autant moins que le comportement spontané de ces citoyens de l'U.R.S.S. faisait paraître la suspicion dont ils étaient l'objet insultante et odieuse. (Thorez, 1982)

Notons que, dans le cas d'une lecture co-variationnelle, le rapport proportionnel implique la réduction du premier procès mais l'accroissement du second : «plus le comportement spontané de ces citoyens de l'U.R.S.S. faisait paraître la suspicion, moins je riais », aspect qui mériterait une étude plus approfondie sur corpus. 


\section{D'autant (Mscal) (x) (que) : locution lexicalisée ou grammaticalisée?}

La séquence en d'autant est affectée par une grande variation intrasystémique, tant au niveau morphologique, syntaxique que sémantique. La multiplicité de ses réalisations pourrait constituer un premier argument pour réfuter d'éventuelles hypothèses à propos de mécanismes de lexicalisation ou de grammaticalisation. En effet, pour devenir plus lexicalisé, un mot ou une locution doit se simplifier, c'est-à-dire perdre l'autonomie de ses constituants internes et donner lieu à « l'entrée d'une nouvelle forme dans le lexique » (cf. Prévost et Fagard 2007 : 4), ce qui n'est manifestement pas le cas d'autant (Mscal) que, structure dans laquelle le second marqueur scalaire est facultatif et variable (plus, moins, mieux). De la même façon, pour avoir subi une grammaticalisation, d'autant (Mscal) que aurait dû faire preuve d'une perte d'autonomie, en endossant un fonctionnement plus grammatical (cf. Brinton et Traugott 2005 : 96-99).

Pourtant, nous sommes d'avis que la séquence d'autant (Mscal) $q u e$, en dépit de ses réalisations multiples, est touchée par des changements importants impliquant une « composante réductrice » (Lehmann 2002). Tout d'abord, autant est toujours précédé par la préposition de, dans sa forme contractée $d$ ', préposition qui a changé de « locatif d'origine » en un marqueur de «mesure ». De ce point de vue, on pourrait dire qu'un processus de grammaticalisation a touché le noyau d'autant dans d'autant (Mscal) que. Deuxièmement, nous avons observé que

- co-variation : causalité emphatique ou contrastée (prédicat non scalaire dans $Q$ )

Drifter ne savait rien de tout cela. Il le savait d'autant moins qu'elle dissimulait tout, et jouait, avec talent, la comédie de l'extase, du bonheur céleste, des yeux qui chavirent et de la pâmoison. (Labro, 1982)

b. portée exophrastique :-co-variation ; causalité scalaire, subjective avec intersubjectification

[...] on ne devrait jamais manifester sur une autoroute. Et d'autant moins que ces dernières, en rase campagne, offrent peu d'échappatoires en cas de charge de la partie adverse. (Rolin, 1996) 
ses divers constituants, dans la forme étendue, apparaissent facilement dans une structure contiguë dont la connotation comparative s'efface en faveur d'une relation causale ; d'autant Mscal que glisse vers l'expression d'une causalité archétypale avec une perte quasi entière de la valeur comparative. Ces tendances semblent répondre aux critères de la lexicalisation. En outre, en tant que conjonction complexe, d'autant (Mscal) que exerce souvent une portée exophrastique, la fonction du second marqueur scalaire s'effaçant dans la matrice à la suite d'un changement de portée. Nous pourrions déceler ici un deuxième processus de grammaticalisation qui succède à la lexicalisation précédente.

Ainsi, d'autant (Mscal) que semble bel et bien concerné par des phénomènes de grammaticalisation et de lexicalisation, qui se manifestent à travers des glissements sémantiques, évoluant de la comparaison à la causalité et s'accompagnant d'un affaiblissement de la portée syntaxique de d'autant plus dans la phrase matrice pour aboutir à un figement progressif de la locution utilisée de manière contiguë à l'initiale de la sous-phrase. Les changements observés pour d'autant (Mscal) que sont en accord avec les étapes reconnues par Traugott (1995) pour la grammaticalisation d'introducteurs de propositions : de la portée endophrastique à la portée exophrastique, avec une perte d'autonomie des constituants de la locution et avec des glissements sémantiques. De plus, la séquence est touchée par l'intersubjectification, avec un changement de la prise en compte du seul point de vue du locuteur (projection scalaire) en une prise en considération de l'interlocuteur, dans les cas où le rapport établi s'approche de la cause contrastée, emphatique ou privilégiée avec une intention claire d'agir sur cet interlocuteur.

Etant donné la grande complexité interne de d'autant (Mscal) que, les mécanismes de changement linguistique se déroulent lentement, progressivement et pas toujours linéairement, ce qui explique la coexistence des différentes réalisations en français d'aujourd'hui. La lexicalisation et/ou la grammaticalisation ne sont pas encore abouties, d'où la variabilité intrasystémique de d'autant (Mscal) que, qui devient un lieu idéal pour observer les divers processus évolutifs en cours. Le plus important de ces processus est, selon nous, celui de la réanalyse, que nous définissons à la suite de Hadermann et al. (à par.) comme : 
[...] une modification de la structure linguistique pas encore exprimée par un réaménagement sur le plan de la construction en surface mais par une instabilité de micro-système, une concurrence d'interprétations possibles qui requièrent de la construction qu'elle se réorganise également en fonction, et conduisent, éventuellement, à une grammaticalisation. La réanalyse serait, dès lors, un point de vue synchronique posé sur une structure dynamique et polyvalente dont le cheminement pourrait être décrit dans une perspective diachronique, s'il y a lieu, en termes de grammaticalisation. ${ }^{13}$

La réanalyse s'observe le mieux dans la forme étendue de la séquence et plus précisément dans les cas où le second marqueur scalaire n'est plus considéré comme ayant une fonction dans la phrase matrice mais comme formant, avec d'autant ... que, une locution insécable utilisée pour introduire une sous-phrase causale. En revanche, dans les emplois non contigus de d'autant Mscal $\boldsymbol{x}$ que, le statut morphosyntaxique et le sémantisme originels de la séquence (et de ses éléments constitutifs) restent présents.

\section{Conclusion}

Il y a donc lieu de distinguer pour d'autant (Mscal) que entre les pôles de « l'immédiat » et de « la distance » qui privilégient respectivement

13 Cette définition s'inspire de celle donnée originellement par Langacker (1977: 58) pour reanalysis :

« [a] change in the structure of an expression or class of expressions that does not involve any immediate or intrinsic modification of its surface manifestation ».

Il existe d'autres appréhensions, telles que celle de Detges et Waltereit (2002) pour qui la réanalyse est un mécanisme opéré par l'interlocuteur lors du décodage d'énoncés, contrairement à la grammaticalisation qui impliquerait plutôt le niveau du locuteur:

«We will show reanalysis to be essentially a hearer-based procedure. Listeners aim at understanding what speakers say. Grammaticalization, on the other hand, is a speaker based phenomenon. Speakers invent expressive discourse techniques in order to pay tribute to basic communicative needs [...]» (Detges et Waltereit, $2002: 151$ ). 
l'innovation et la stabilité (Koch et Oesterreicher 2001). Pour notre séquence, il existe une tension entre ces deux pôles : d'une part, elle semble résister aux tendances évolutives et maintenir ses valeurs de base dans un certain nombre d'emplois ; d'autre part, caractéristique surtout du registre argumentatif avec une nette orientation intersubjective, elle tend aussi à se renouveler et à véhiculer de nouvelles acceptions, moins comparatives. La réanalyse permet d'expliquer cette tension ainsi que la variabilité sémantique et morphosyntaxique qui en résulte. D'autant (Mscal) que est en train de se modifier, ce qui se manifeste à travers la coexistence d'interprétations et de fonctionnements aboutissant sans doute à une séquence plus grammaticalisée et aussi plus lexicalisée. La coexistence de valeurs ne permet toutefois pas encore de prédire avec certitude quels emplois seront privilégiés au détriment de quels autres fonctionnements. Mais, ce qui est clair, c'est que dans le cas de d'autant Mscal que les mécanismes évolutifs ne s'opèrent pas indépendamment, comme le montre le tableau suivant.

Tableau 3 : emplois de d'autant Mscal que.




Pour terminer, ajoutons encore que les corpus se révèlent très utiles pour mieux cerner et expliquer la variabilité d'une occurrence : ils permettent non seulement d'étudier les fréquences mais également d'analyser les emplois par le biais de la prise en compte entre autres de paramètres contextuels (la nature scalaire ou non des prédications liées dans notre étude). Après avoir identifié les configurations possibles pour d'autant (Mscal) que et leurs traits distinctifs pertinents, il sera intéressant de vérifier ultérieurement comment celles-ci se répartissent statistiquement afin de dévoiler les tendances préférentielles du français moderne et d'approfondir l'influence des paramètres « dia », par exemple le diaphasique. Une telle étude se révélera aussi indispensable pour alimenter les ouvrages de référence et pour affiner la description du fonctionnement de séquences complexes comme d'autant (Mscal) que.

\section{Références}

Athanasiadou, A., 2007, « On the subjectivity of intensifiers », Language Sciences, 29, p. 554-565.

Beck, S., 1997, « On the semantics of comparative conditionals », Linguistics and Philosophy, 20, p. 229-271.

Bertocchi, A. et M. Maraldi, 2010, « Latin comparative correlatives and scalarity », in : P. Hadermann et O. Inkova (éds), Approches de la scalarité, Genève : Droz, p. 88-105.

Brinton, L. J. et E. C. Traugott, 2005, Lexicalization and Language Change, Cambridge : Cambridge University Press.

Degand, L. et B. Fagard, 2008, « (Inter)subjectification des connecteurs : le cas de car et parce que », Revista de Estudos Linguísticos da Universidade do Porto, 3, p. 119-136.

Detges, U. et R. Waltereit, 2002, « Reanalysis vs. Grammaticalization : a Semantic-Pragmatic Account of Functional Change in Grammar », in : Zeitschrift für Sprachwissenschaft, 21, p. 151-195. 
Dict. Acad. Fr. = Dictionnaire de l'Académie française (<http://atilf. atilf.fr/academie9.htm $>$ ).

Frantext - base textuelle ( $<$ www.frantext.fr $/>$ ).

Fournier, N., C. Fuchs et P. Le Goffic, 2008, « Structures à subordonnée comparative en français : problèmes de représentation syntaxique et sémantique », Linguisticae Investigationes, 31 : 1, p. 11-61.

Fuchs, C. et N. Fournier, 2007, «Que et comme marqueurs de comparaison », Lexique, 18, p. 69-107.

Giacalone Ramat, A. et P. Hopper, (éds), 1998, The Limits of grammaticalization, Amsterdam : Benjamins.

Grevisse, M. et A. Goosse, 2011, Le bon usage, Bruxelles : De Boeck-Duculot, 15 ème éd.

Guimier, Cl., 1996, Les adverbes du français : le cas des adverbes en -ment, Paris : Ophrys.

Hadermann, P., à par., « D'autant plus que et la corrélation en français ».

Hadermann, P. et O. Inkova, 2010, Approches de la scalarité, Genève : Droz.

Hadermann, P., M. Pierrard, D. Van Raemdonck et V. Wielemans, 2010, « Les structures corrélatives : pour une inscription dans les sous-systèmes parataxe/hypotaxe et coordination/subordination », in : M.-.J. Béguelin, M. Avanzi et G. Corminboeuf (éds), La parataxe, tome 2 : structures, marquages et exploitations discursives, Bern : Peter Lang, p. 219-240.

Hadermann, P., Meinertzhagen, L., Pierrard, M., Roig, A. et D. Van Raemdonck, (à par.), « Pour une réanalyse catégorielle et fonctionnelle de la construction $X$ de même que $Y$ ».

Hopper, P. J. et E. C. Traugott, 2003, Grammaticalization, Cambridge : Cambridge University Press.

Inkova, O. et P. Hadermann, 2013, La corrélation. Aspects syntaxiques et sémantiques, Genève : Droz.

Koch, P. et W. Oesterreicher, 2001, « Langage parlé et langage écrit », in : G. Holtus, M. Metzeltin et C. Schmitt (éds), Lexikon der Romanistischen Linguistik, tome I/2, Tübingen : Niemeyer, p. 584-627. 
Kuyumcuyan, A., 2008, « Les modifieurs de déclencheurs dans les systèmes corrélatifs », Lingvisticce Investigationes, 31 : 1, p. 107-123. Langacker, R.W., 1977, "Syntactic Reanalysis », in : Ch. Li (éd.), Mechanisms of Syntactic Change, Austin : University of Texas Press, p. 57-139.

Le Goffic, P., 1993, Grammaire de la phrase française, Paris : Hachette. Lehmann, Ch., 2002, "New reflections on grammaticalization and lexicalization », in : I. Wischer et G. Diewald (éds), New Reflections on Grammaticalization - Proceedings from the International Symposium on Grammaticalization, 17-19 June 1999, Potsdam, Germany, Amsterdam/Philadelphia : Benjamins, p. 1-18.

Petit Robert $=$ Robert, P., 2008, Le Nouveau Petit Robert, version électronique, Paris : Editions Le Robert.

Prévost, S. et B. Fagard, 2007, « Grammaticalisation et lexicalisation : la formation d'expressions complexes », Langue française, 156, p. 3-8. Sanchez Lopez, C., 2010, « Scalarité et corrélation : syntaxe et sémantique des corrélatives comparatives en espagnol », in : P. Hadermann et $\mathrm{O}$. Inkova (éds), Approches de la scalarité, Genève : Droz, p. 106-132. Traugott, E. C., 1989, « On the rise of epistemic meanings in English : an example of subjectification in semantic change », Language, 65, p. 31-55.

Traugott, E. C., 1994, « Grammaticalization and lexicalization », in : R. Asher et J. Simpson (éds), Encyclopedia of Language and Linguistics, vol 3, Oxford : Pergamon, p. 1481-1486.

Traugott, E. C., 1995, « The role of the development of discourse markers in a theory of grammaticalization », Paper presented at the International Conference of Historical Linguistics XII, Manchester ( $<$ http://www.stanford.edu/ traugott/papers/discourse.pdf $>$ ).

Traugott, E. C., 2003, « From subjectification to intersubjectification », in : R. Hickey (éd.), Motives for Language Change, Cambridge : Cambridge University Press, p. 124-139.

Traugott, E. C. et R. B. Dasher, 2002, Regularity in semantic change, Cambridge : Cambridge University Press.

$T L F i=$ Trésor de la Langue Française informatisé (<http://atilf.atilf.fr/>). 